

\title{
UM ESTUDO SOBRE USO DO OPEN BANKING ENTRE UNIVERSITÁRIOS NA GRANDE SÃO PAULO UTILIZANDO O MODELO DE PRONTIDÃO E ADOÇÃO DE TECNOLOGIA (TRAM)
}

\author{
An analysis of Open Banking adoption among university students in São Paulo \\ using the Technology Readiness and Acceptance Model (TRAM)
}

Heitor Valarini ${ }^{1}$

(iD)

Davi Nakano²

\author{
${ }^{1}$ Engenheiro \\ Escola Politécnica, Universidade de São Paulo \\ São Paulo, SP - Brasil. \\ heitor.valarini@usp.br \\ 2 Doutor \\ Escola Politécnica, Universidade de São Paulo \\ São Paulo, SP - Brasil. \\ dnnakano@usp.br
}

Recebido em: 16 jun. 2020

Aprovado em: 06 nov. 2020
Resumo: O compartilhamento de dados bancários de clientes entre as instituições financeiras é chamado de Open Banking. Ele permite a consolidação das informações de diferentes contas bancárias em um único local e a realização de transferências e pagamentos sem a necessidade de acesso a um banco específico. Sua adoção visa o aumento da competição e eficiência no mercado financeiro, e atualmente está em regulamentação pelo Banco Central do Brasil. Este artigo utiliza o Technology Readiness and Acceptance Model (TRAM) para avaliar a intenção de uso do Open Banking entre jovens universitários. Os resultados obtidos indicam que a utilidade percebida é afetada pelo otimismo, desconforto e insegurança, enquanto a facilidade de uso percebida é afetada somente pela inovatividade. Dado que os modelos de aceitação de tecnologia são sensíveis ao contexto cultural de cada país, este estudo contribui para a literatura, e oferece uma visão do potencial de adoção do Open Banking entre a parcela da população que será grande usuária dessa tecnologia.

Palavras-chave: Prontidão tecnológica. Adoção de tecnologia. Open Banking. Tecnologia bancária.

Abstract: Open Banking refers to the sharing of customer bank data between financial institutions. It allows consolidating data from different bank accounts in one place, and money transfers and payments without accessing a specific bank. Its adoption aims at increasing competition and efficiency in the financial market, and the Central Bank of Brazil is currently setting norms for its application. This article applies the Technology Readiness and Acceptance Model (TRAM) to assess Open Banking adoption among university students. The results indicate that Perceived Usefulness is affected by Optimism, Discomfort and Insecurity, while Perceived Ease of Use is affected only by Innovativeness. Given that the technology acceptance models are culture and national context sensitive, this study contributes to the literature, and offers an overview of the potential for Open Banking adoption among the cohort that will likely to be the major user of that technology.

Key words: Technology readiness. Technology acceptance. Open Banking. Financial technology. 


\section{Introdução}

O compartilhamento de dados bancários de clientes entre as instituições financeiras através de interfaces de programação de aplicativos (API) é chamado de Open Banking. Ele permite, por exemplo, a consolidação das informações de diferentes contas bancárias em um único local, e a realização de transferências e pagamentos sem a necessidade de acesso a um banco específico. Sua adoção visa a entrada de novos agentes no mercado financeiro e o aumento da competição, e consequentemente, da eficiência do setor, e atualmente está em regulamentação pelo Banco Central do Brasil. A iniciativa, porém, tem encontrado resistência em países onde sua adoção está em estágio mais avançado (Thomas et al., 2019).

De fato, embora a tecnologia digital tenha se tornado praticamente indispensável tanto na vida profissional quanto na pessoal, sua adoção encontrou, e encontra, resistência por parte das pessoas. Para entender esse comportamento e suas causas, diversos modelos foram desenvolvidos. Dentre esses, um dos mais conhecidos é o de Davis (1989), o Technology Acceptance Model (TAM), que relaciona a adoção da tecnologia por uma pessoa à sua percepção da utilidade e facilidade de uso. Outro conceito bastante utilizado é o de prontidão tecnológica, que avalia o quanto uma pessoa é propensa a adotar novas tecnologias em sua vida pessoal e profissional. Ele avalia as dimensões de otimismo, inovatividade, desconforto e insegurança, e foi operacionalizado em um índice, o Technology Readiness Index (TRI) (Parasuraman, 2000; Parasuraman \& Colby, 2015). Os dois modelos foram conjugados no Technology Readiness and Acceptance Model (TRAM), que utiliza as dimensões de prontidão tecnológica para avaliar a percepção de utilidade e facilidade de uso individual (Lin et al., 2007).

Este trabalho utiliza o TRAM para analisar a adoção do Open Banking entre jovens universitários de 18 e 30 anos, a chamada geração "nativa digital", mais habituada e aberta à novas tecnologias. Avaliar o grau de propensão ao uso do Open Banking por esses jovens pode dar uma visão do prospecto da adoção da iniciativa no país. Embora outros estudos já tenham analisado a adoção de tecnologias bancárias em outros países (Shin \& Lee, 2014; Sivathanu, 2019), a literatura indica que a cultura e contexto locais influenciam na propensão à adoção de uma tecnologia (Im et al., 2011), o que justifica este estudo.

Utilizando um questionário adaptado de Lin et al. (2007), foram obtidas 169 respostas de jovens universitários na Grande São Paulo. Os resultados obtidos indicam que a utilidade percebida é afetada pelo otimismo, desconforto e insegurança, enquanto a facilidade de uso percebida é afetada somente pela inovatividade, o que contraria resultados da literatura. Isso sugere que as dimensões do TRI afetam de forma diferente a percepção de tecnologias já disponíveis com as que ainda não foram lançadas, que é o caso do Open Banking no Brasil. Este artigo apresenta duas contribuições: do ponto de vista 
acadêmico, os modelos de aceitação de tecnologia são sensíveis ao contexto cultural de cada país, e existem poucos estudos tanto de aceitação de tecnologia quanto de Open Banking no Brasil. Seus resultados também sugerem que a análise da adoção deve considerar a disponibilidade da tecnologia como fator moderador das relações do TRAM. Do ponto de vista profissional, o estudo traz uma visão do potencial de adoção do Open Banking entre a parcela da população que será uma grande usuária dessa tecnologia.

\section{Modelos de adoção de novas tecnologias}

Diversos modelos foram desenvolvidos para explicar a adoção da TI pelas pessoas. Eles procuram estudar os determinantes da intenção de uso, que posteriormente levam à sua utilização (Venkatesh et al., 2003). Entre esses modelos, um dos mais conhecidos é o modelo de aceitação da tecnologia, ou Technology Acceptance Model (TAM) (Davis, 1989), que busca explicar a adoção de uma tecnologia por indivíduos em seu ambiente de trabalho. Ele é baseado na Teoria da ação racional, que preconiza que o comportamento individual é resultado de uma intenção para a ação, construída a partir de crenças, atitudes e pressupostos normativos (Legris et al., 2003). De acordo com o modelo, dois fatores são determinantes na aceitação de uma nova tecnologia por um usuário: a utilidade percebida, o grau em que um indivíduo acredita que o uso da tecnologia aumenta seu desempenho no trabalho, e a facilidade de uso percebida, o grau em que um indivíduo acredita que o seu uso será livre de esforço. Ambas influenciam a atitude que uma pessoa terá perante uma determinada tecnologia, e não são independentes: a facilidade de uso percebida afeta diretamente a utilidade percebida.

A prontidão tecnológica, ou Technology Readiness, é definida como a propensão individual para adoção de uma nova tecnologia, que é influenciada por suas percepções, crenças, sentimentos e motivações (Lam et al., 2008), e ao contrário do TAM, não procura avaliar a adoção individual da TI, mas qual a imagem, ou perspectiva, que uma pessoa tem sobre ela. Essa visão pode variar de amplamente favorável a amplamente desfavorável, o que vai, evidentemente, influenciar a adoção. Os fatores motivadores e inibidores da prontidão tecnológica são classificados em quatro dimensões (Parasuraman, 2000):

- Otimismo: Visão positiva em relação à tecnologia, reflete a crença de que ela propicia aos indivíduos maior controle, flexibilidade e eficiência nas suas vidas.

- Inovatividade: Tendência do indivíduo a ser pioneiro na adoção de tecnologia ou líder de opinião, ou quanto indivíduos entendem a si mesmos como protagonistas na adoção tecnológica. 
- Desconforto: Percepção de insuficiência de controle sobre a tecnologia e o sentimento de ser oprimido por ela, o medo e as preocupações das pessoas em relação à tecnologia.

- Insegurança: Desconfiança e ceticismo com relação ao correto funcionamento da tecnologia e do medo de que ela tenha consequências danosas.

Otimismo e Inovatividade são motivadores da prontidão tecnológica, contribuindo para a maior propensão de adoção, enquanto desconforto e insegurança atuam como seus inibidores. Os quatro fatores foram operacionalizados em escalas, gerando o índice Technology Readiness Index (TRI) (Parasuraman, 2000; Parasuraman \& Colby, 2015). Resultados utilizando o TRI mostraram que as quatro dimensões atuam de modo independente, contribuindo de maneira única para o entendimento da prontidão à tecnologia pelo indivíduo (Parasuraman, 2000).

O TAM foi desenvolvido para avaliar a adoção da TI no ambiente de trabalho (Rojas-Méndez et al., 2017), onde a escolha individual é feita dentro de restrições impostas pela organização, o que limita a sua aplicação fora desse contexto (Lin et al., 2007). Já o TRI estima a propensão individual ao uso da tecnologia, em qualquer ambiente. Considerando que seja razoável admitir que a postura do indivíduo em relação à tecnologia em geral influencia a sua percepção quanto à utilidade e facilidade de uso de tecnologias específicas, O TRI pode ser combinado ao TAM para avaliar a adoção da TI em situações extra-profissionais (Lin et al., 2007). Dessa forma, no modelo de prontidão e aceitação à tecnologia, ou Technology Readiness and Acceptance Model (TRAM), os quatro construtos do TRI são considerados determinantes das dimensões do TAM (Lin et al., 2007). Dentro desse raciocínio, otimismo e inovatividade levam à maior percepção de utilidade e facilidade de uso, enquanto insegurança e desconforto inibem as mesmas dimensões. O TRAM foi utilizado na avaliação de diversas novas tecnologias: o NFC na Coréia (Shin \& Lee, 2014), um sistema de negociação de ações em Taiwan (Lin et al., 2007) e a tecnologia móvel de informação na saúde também em Taiwan (Kuo et al., 2013).

A literatura aponta que há diferenças na aceitação de uma tecnologia entre países, devido à características de seu setor produtivo e de seus mercados, assim como das políticas públicas relacionadas à tecnologia (Im et al., 2011). A própria cultura local influencia o comportamento individual, e foi apontada como fator de influência na aceitação de uma tecnologia (Sun et al., 2019). Assim, resultados da aceitação de uma tecnologia em um país não são necessariamente extensíveis a outros. Embora a adoção de tecnologias bancárias já tenham sido avaliados em outros países com o uso do TRAM (Sivathanu, 2019), ainda existem pouco estudos no contexto brasileiro. 


\section{0 setor bancário brasileiro}

Desde 1994, com a implementação do Plano Real, ocorreram uma série de fusões e aquisições no setor bancário brasileiro, o que levou uma redução no número de bancos no país: dos 242 ativos em 1995, haviam 156 em 2007, e 151 em 2018 (Banco Central, 2019). Além da tendência de redução no número de instituições bancárias, o mercado de crédito brasileiro apresenta alto grau de concentração: segundo o Relatório de Economia Bancária publicado pelo Banco Central em 2018, os 5 principais bancos que operam no Brasil (Itaú, Bradesco, Banco do Brasil, Santander e Caixa Econômica Federal) concentraram $84,8 \%$ das operações de crédito concedidas no país.

A baixa concorrência tem influência no custo do crédito (BACEN, 2019), fazendo o spread bancário brasileiro ser um dos maiores do mundo (FEBRABAN, 2017). Em 2016, ele era de 22\%, valor muito superior a outros países da América Latina, como México $(9,1 \%)$ e Chile $(4,3 \%)$, e do restante do mundo, como Alemanha (5,5\%), Estados Unidos (5,2\%) e Índia (4,0\%). A redução do spread bancário passa pelo aumento da competição e da eficiência operacional para reduzir os custos associados à oferta de crédito (FEBRABAN 2017).

Entre as principais tendências observadas no setor bancário brasileiro, destaca-se a adesão da população ao internet banking e às tecnologias bancárias ofertadas por empresas emergentes de tecnologia financeira, as fintechs. Intensificou-se, por exemplo, o interesse no mercado pelos bancos digitais, devido à maior flexibilidade e capacidade de atendimento de necessidades dos clientes que oferecem. Segundo pesquisa do Serviço de Proteção de Crédito (SPC), os bancos digitais atraem principalmente o público mais jovem, que busca a isenção de anuidade, e taxas mais baixas em relação aos bancos tradicionais.

Em vista do contexto atual do setor bancário brasileiro, o Banco Central lançou, em 2019, a Agenda BC\#. Ela contém os principais pilares que nortearão as ações da autoridade monetária nos próximos anos, e está estruturada em quatro dimensões: inclusão, transparência, educação e competitividade. A última dimensão abrange iniciativas que fomentem o aumento da competição, reduzam os juros cobrados e ampliem o acesso da população aos serviços financeiros. O surgimento das fintechs aumenta a competição no mercado e alinha-se com a Agenda BC\#, na qual o Open Banking figura como iniciativa, pois pode impulsionar a criação de novas empresas e serviços.

\section{0 open banking}

O Open Banking, ou Sistema Financeiro Aberto, é o compartilhamento de dados padronizados de clientes entre instituições financeiras e outras instituições autorizadas pelo Banco Central, por meio da integração de plataformas e infraestrutura de sistemas de informação. Ele se baseia na premissa de que os dados pertencem a seus consumidores, que mediante seu consentimento, são compartilhados 
entre instituições, permitindo maior oferta de produtos e serviços financeiros. A figura 1 apresenta o modelo do Open Banking.

Figura 1

Modelo do Open Banking

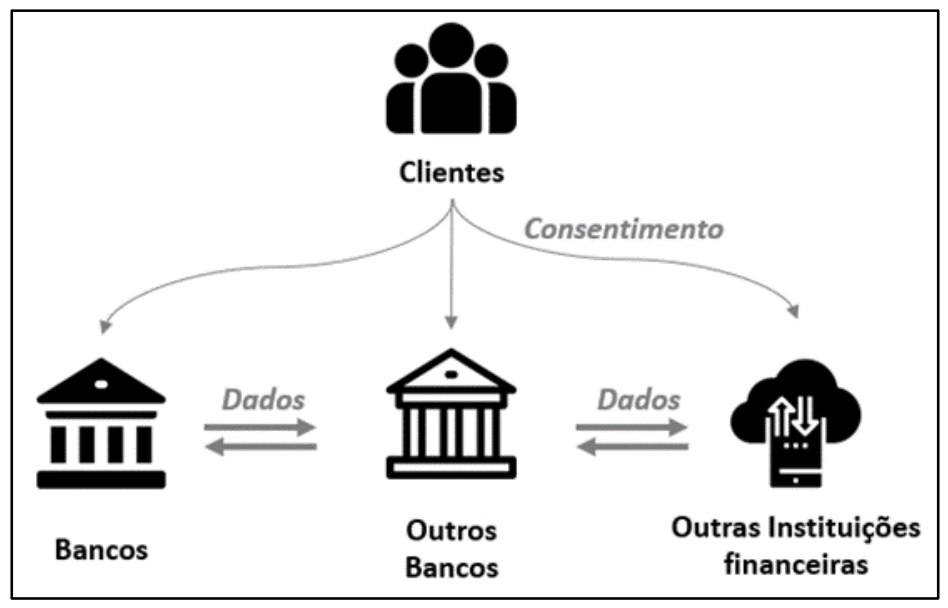

Fonte: Os autores.

O Open Banking favorece a comparação entre produtos e serviços disponíveis no sistema financeiro, contribuindo para a criação de um ambiente mais competitivo e eficiente na oferta de serviços financeiros, crédito e meios de pagamento. Nos países europeus onde a iniciativa foi implementada, a regulamentação estabeleceu dois novos tipos de prestadores de serviços: os provedores de serviços de informação de conta (Account Information Service Providers - AISPs) e os prestadores de serviços de iniciação de pagamento (Payment Initiation Service Provider - PISPs). Os AISPs permitem a visualização do extrato consolidado das contas bancárias do consumidor em um único aplicativo, independentemente da instituição financeira onde estão localizadas. Os PISPs possibilitam a realização de transferências e realização de pagamentos de contas sem a necessidade de acesso direto a sites ou aplicativos de bancos.

O Open Banking já foi implementado, ou está em implementação, no Reino Unido, União Europeia e Austrália, impulsionado por motivação semelhante à brasileira: fomento a competição e a inovação no setor. Porém, segundo um estudo sobre confiança do consumidor e o ambiente regulatório do Open Banking em dez países publicado em 2019 (Thomas et al., 2019), a confiança dos consumidores na iniciativa se mantém baixa, mesmo com o estímulo proporcionado pelo ambiente regulatório (figura 2). A relutância por parte dos consumidores em compartilhar seus dados bancários pode ser parcialmente explicada por uma mentalidade de desconfiança em relação aos bancos, que pode variar de acordo com o país e sua a cultura, e pela insegurança provocada por diversos episódios de vazamentos de dados ocorridos em empresas e plataformas digitais no mundo. 
Figura 2

Regulação, confiança e sentimento do consumidor

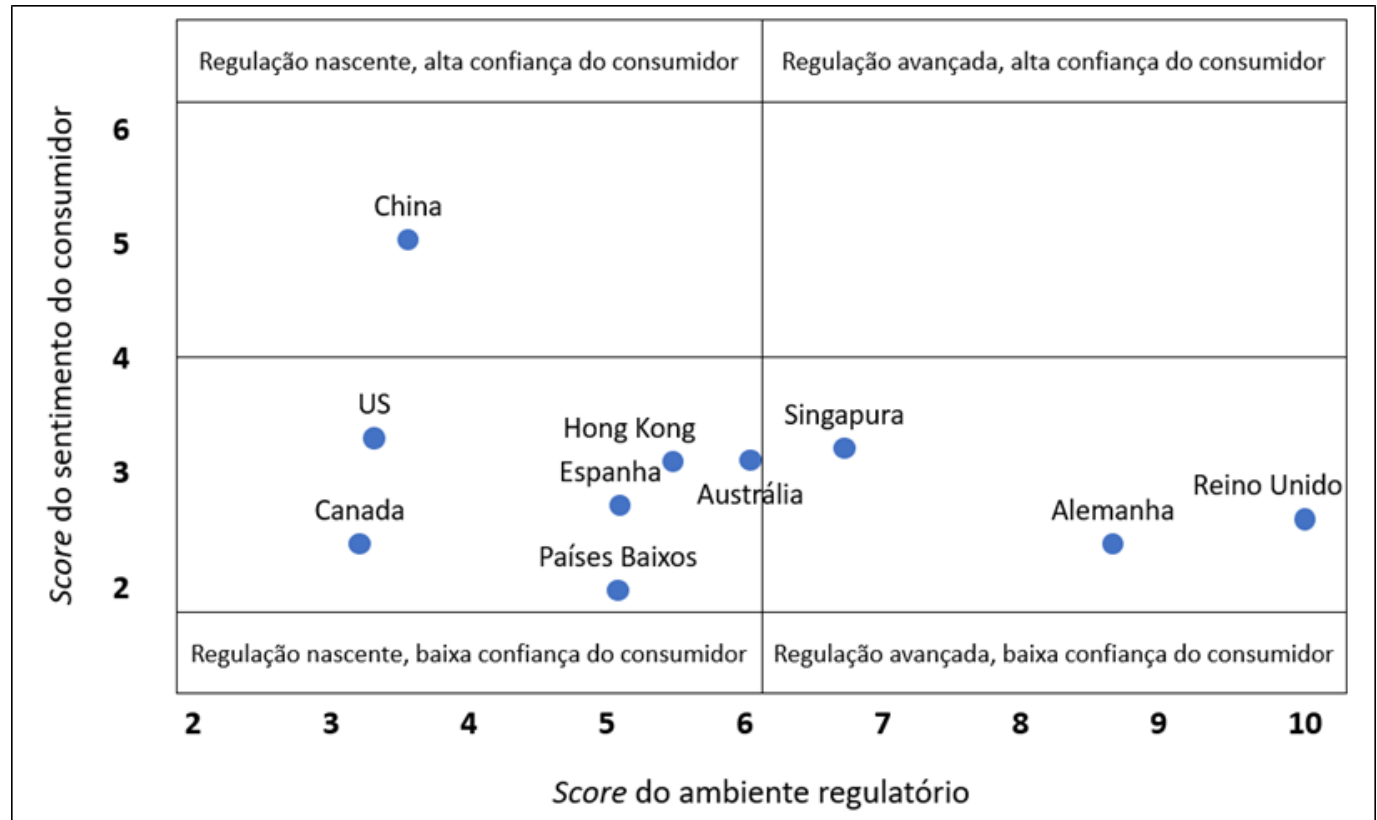

Fonte: adaptado de Thomas et al. (2019).

A implantação do Open Banking é um dos principais focos do Banco Central. Em abril de 2019, o agente regulador publicou o Comunicado 33.455, que estabeleceu diretrizes e cronograma de implementação. Em janeiro de 2020, o Banco Central finalizou o processo de consulta pública da proposta de regulamentação, visando definir escopo mínimo das instituições participantes, requisitos para participação, dados e serviço contemplados pelo Open Banking, entre outros aspectos. A implementação em si é composta por quatro fases: a primeira está prevista para o segundo semestre de 2020 e deve abranger o compartilhamento de dados de produtos e serviços pelas instituições. As fases posteriores consistem no compartilhamento de dados cadastrais e transações dos clientes, serviços prestados e de outros produtos financeiros, como investimentos e seguros.

\section{Uma análise da adoção do Open Banking no Brasil}

A adoção de tecnologias bancárias foi analisada por diversos estudos. Utilizando o TRAM, a adoção de pagamentos utilizando smartphones foi analisada por Shin \& Lee (2014) na Coréia do Sul, Acheampong et al. (2017) estudaram a adoção de sistemas de pagamento eletrônico em Gana, e Sivathanu (2019) o Open Banking na Índia. A sensibilidade do TRAM ao contexto e cultura locais já foi indicada como importante (Im et al., 2011), e as dimensões do TRI foram consideradas dependentes da cultura e do nível de desenvolvimento tecnológico do país (Ramírez-correa et al., 2020). Dessa forma, embora o estudo de Sivathanu (2019) já tenha analisado o Open Banking, considerando a diferença no 
nível de penetração da internet no Brasil e na Índia, 70\% e 40\% respectivamente, (Internetworldstats, 2020), e a possível influência do contexto e cultura locais, um estudo baseado na aplicação do TRAM no Brasil é relevante para o entendimento da propensão da adoção do Open Banking o país.

A utilização do TRAM na adoção de tecnologias já foi feita por diversos estudos (Acheampong et al., 2017; Kuo et al., 2013; Lin et al., 2007; Shin \& Lee, 2014; Sivathanu, 2019; Tsikriktsis, 2004). As seguintes relações foram apontadas: pessoas que apresentam Otimismo (OTM) em relação à tecnologia, ou apresentam Inovatividade (INO), geralmente possuem uma atitude positiva ao seu uso, levando à Facilidade de Uso Percebida (FUP) e à Utilidade Percebida (UPC). Desta forma, as seguintes hipóteses podem ser formuladas:

H1: OTM influencia positivamente a UPC para a tecnologia do Open Banking

H2: OTM influencia positivamente a FUP para a tecnologia do Open Banking

H3: INO influencia positivamente a UPC para a tecnologia do Open Banking

H4: INO influencia positivamente a FUP para a tecnologia do Open Banking

Os mesmos estudos apontam que Desconforto (DCF) e Insegurança (ISG) são aspectos negativos em relação à percepção de facilidade e utilidade, e por consequência, da intenção de uso de uma tecnologia. Portanto pode-se formular as seguintes hipóteses:

H5: DFC influencia negativamente a UPC para a tecnologia do Open Banking H6: DFC influencia negativamente a FUP para a tecnologia do Open Banking H7: ISG influencia negativamente a UPC para a tecnologia do Open Banking H8: ISG influencia negativamente a FUP para a tecnologia do Open Banking

Por fim, existem ainda a relação entre a Facilidade de uso percebida (FUP) e a Utilidade percebida (UPC) (Davis, 1989). Assim, é proposta a seguinte hipótese:

H9: FUP influencia positivamente a UPC para a tecnologia do Open Banking

Finalmente, o TAM sugere que os fatores de Utilidade percebida (UPC) e Facilidade de uso percebida (FUP) atuam positivamente para a previsão da intenção de uso para uma nova tecnologia (Davis, 1989), neste caso, o Open Banking. Assim chamando a intenção de uso de IOB, temos:

H10: UPC influencia positivamente a IOB

H11: FUP influencia positivamente a IOB

A figura 3 ilustra o modelo TRAM e as hipóteses formuladas. 
Figura 3

Modelo TRAM e hipóteses

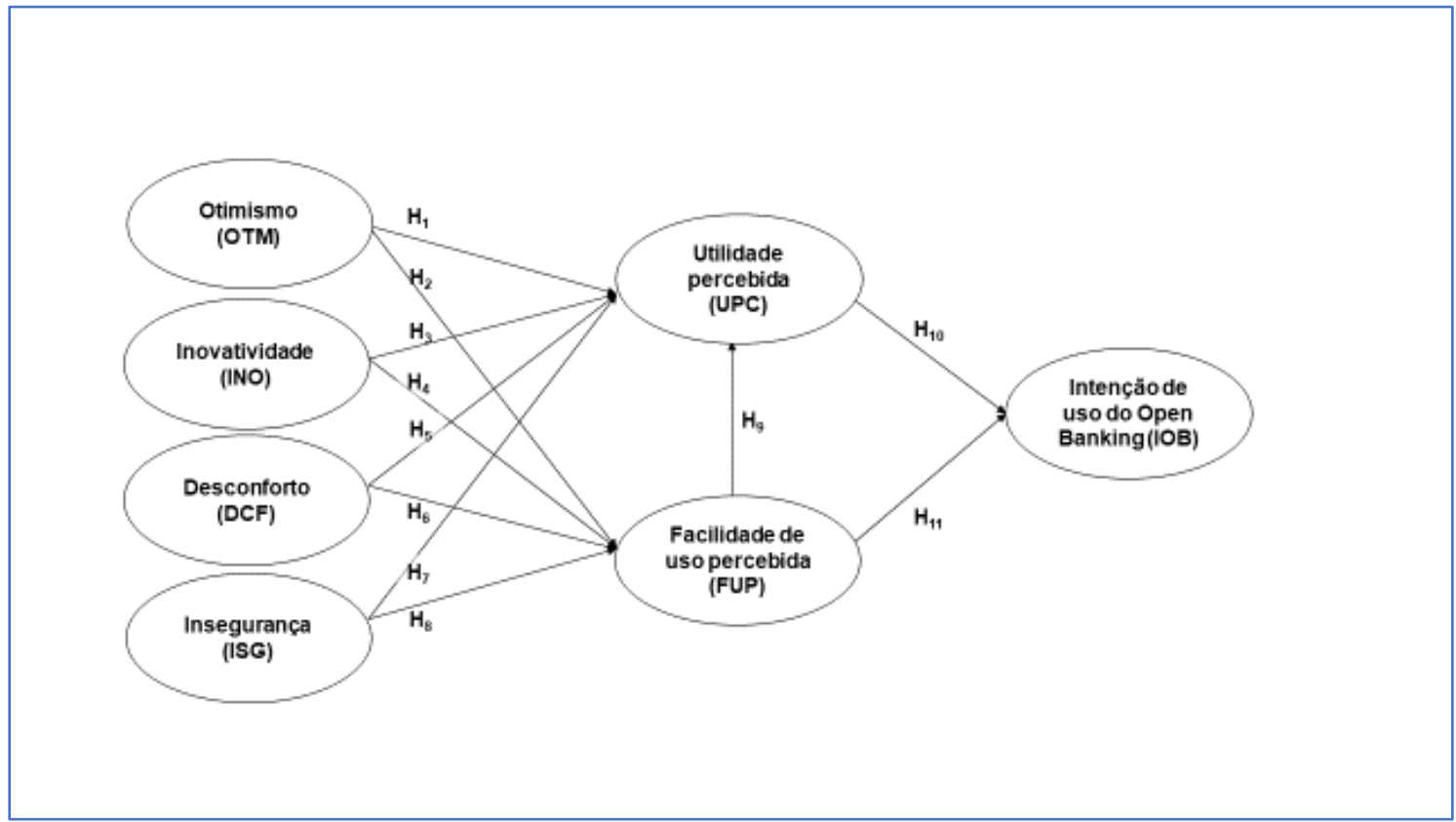

Fonte: Os autores.

\section{Método de pesquisa}

Para a verificação das hipóteses, foi elaborado um questionário baseado em Lin et al. (2007), composto por 40 perguntas, com questões específicas do modelo TRAM, além de outras sobre o perfil dos respondentes, como idade, gênero, grau de escolaridade, a posse de conta bancária e seu uso de tecnologias bancárias. Para esclarecer e orientar os respondentes, o questionário possuía uma seção introdutória onde o conceito do Open Banking e sua aplicação eram brevemente apresentados (O questionário utilizado encontra-se no anexo 1). Um teste piloto foi conduzido com cinco respondentes selecionados, para verificar a clareza da redação e dúvidas no preenchimento das perguntas.

Após os ajustes decorrentes do teste piloto, foi realizada a pesquisa, que utilizou uma amostra não-probabilística composta por jovens universitários com idade entre 18 e 30 anos da região metropolitana de São Paulo, que possuem conta bancárias e fazem uso de internet banking. A escolha desse público teve por objetivo avaliar a propensão para o uso do Open Banking por pessoas que estão familiarizadas ao ambiente digital e que já fazem uso habitual de tecnologias bancárias, procurando evitar, dessa forma, desvios relacionados à pouca familiaridade ou mesmo rejeição ao mundo virtual.

A pesquisa foi realizada entre os dias 27 de fevereiro a 20 de março de 2020. A maior parte das respostas foi coletada de forma presencial, através da abordagem de pessoas em locais de grande fluxo na Cidade Universitária da Universidade de São Paulo, como os restaurantes. Esse método garantiu um bom número de respostas (98 respondentes), porém, devido à pandemia Covid19, teve de ser suspenso. 
O questionário foi então administrado de maneira virtual, utilizando contatos de universitários através de redes sociais (72 respondentes). Foram coletadas 170 respostas no total, das quais 169 foram consideradas válidas para a análise, já que uma das respostas foi descartada pelo fato do respondente não utilizar internet banking.

\section{Análise dos dados}

A análise dos dados foi feita utilizando modelagem por equações estruturais com regressão por mínimos quadrados parciais (PLS-SEM), utilizando o software Smart PLS 3.0. A técnica permite o exame simultâneo de um conjunto de relações teóricas entre variáveis, e é indicada quando se procura identificar variáveis preditoras chave. Ela tem sido aplicada em estudos nos campos da psicologia, sociologia, administração, biologia, entre outros, devido à sua capacidade de: permitir lidar com diversas relações simultaneamente com eficiência estatística, e avaliar as relações de maneira abrangente, fornecendo uma transição da análise exploratória para a confirmatória (Hair et al., 1995).

O modelo de equações estruturais é dividido em modelo de mensuração e modelo estrutural. O primeiro tem por finalidade avaliar a capacidade dos indicadores em medir com precisão o conceito associado às variáveis latentes estabelecidas. Como sugerem Hair et al. (1995), foram avaliadas a validação convergente, a validação discriminante e a análise de confiabilidade.

- Validação convergente: avaliação do grau em que duas medidas do mesmo conceito estão correlacionadas. Ocorre quando a variância média extraída (AVE) para cada variável latente for superior a 0.5 .

- Validação discriminante: avaliação do grau em que uma variável latente é distinta das demais, verificando se os indicadores têm relação mais forte com sua variável latente do que com as demais. A validação discriminante ocorre quando as correlações entre as variáveis latentes forem inferiores à raiz quadrada da AVE de cada uma.

- Análise de confiabilidade: avaliação da consistência interna entre os valores dos indicadores que compõem uma variável latente. Ela é feita através do cálculo do Alfa de Cronbach e da avaliação da confiabilidade composta (CR), sendo que ambos os valores devem ser superiores a 0.7 .

Além disso, os valores das cargas externas (outer loadings) de cada indicador foram verificados. Neste estudo, foram mantidos na análise os indicadores com cargas fatoriais com valores iguais ou superiores a 0.6. 
O modelo estrutural define o conjunto de relações de dependência entre as variáveis latentes. Segundo Hair et al. (1995), o modelo estrutural é útil para representar a inter-relação das variáveis, que é dada através do cálculo dos coeficientes de caminho $(\beta)$, que indicam o efeito direto de uma variável sobre outra. As hipóteses deste estudo e o modelo estrutural utilizado estão apresentados na figura 3.

\section{Resultados}

Os respondentes que compõem a amostra são adeptos e fazem uso frequente das tecnologias utilizadas pelo sistema bancário brasileiro (79\% dos respondentes utilizam o internet banking no mínimo uma vez por semana). Supõe-se que tal público tenha compreensão e propensão a aderir a novas tecnologias bancárias, como o Open Banking. O perfil demográfico dos respondentes está apresentado na Tabela 1.

Tabela 1

Perfil demográfico dos respondentes $(n=169)$

\begin{tabular}{|c|c|c|c|}
\hline Perfil & & $\begin{array}{l}\text { Frequência } \\
\text { absoluta }\end{array}$ & $\begin{array}{l}\text { Frequência } \\
\text { relativa }\end{array}$ \\
\hline \multirow{4}{*}{ Idade } & 18 a 20 anos & 22 & $13 \%$ \\
\hline & 21 a 23 anos & 90 & $53 \%$ \\
\hline & 24 a 26 anos & 51 & $30 \%$ \\
\hline & 27 a 29 anos & 6 & $4 \%$ \\
\hline \multirow{3}{*}{ Gênero } & Feminino & 65 & $38 \%$ \\
\hline & Masculino & 102 & $60 \%$ \\
\hline & Outro & 2 & $1 \%$ \\
\hline \multirow{4}{*}{ Grau de escolaridade } & Até ensino médio completo & 5 & $3 \%$ \\
\hline & Ensino superior incompleto & 107 & $63 \%$ \\
\hline & $\begin{array}{l}\text { Ensino superior completo até } 3 \\
\text { anos }\end{array}$ & 42 & $25 \%$ \\
\hline & $\begin{array}{l}\text { Ensino superior completo há mais } \\
\text { de } 3 \text { anos }\end{array}$ & 15 & $9 \%$ \\
\hline \multirow{4}{*}{$\begin{array}{l}\text { Frequência de uso de internet } \\
\text { banking }\end{array}$} & Menos de uma vez por mês & 9 & $5 \%$ \\
\hline & Uma a duas vezes por mês & 27 & $16 \%$ \\
\hline & Uma a duas vezes por semana & 52 & $31 \%$ \\
\hline & Mais de duas vezes por semana & 81 & $48 \%$ \\
\hline
\end{tabular}

Fonte: Os autores.

A Tabela 2 apresenta os valores calculados para a validação discriminante. Os valores que compõe a diagonal consistem na raiz quadrada da variância média extraída (AVE) da respectiva variável latente, enquanto os demais valores consistem nas correlações entre as diferentes variáveis. Visto que para cada variável latente, a raiz quadrada da AVE é maior que os valores das correlações entre ela e as demais variáveis, verifica-se a validade discriminante. 
A variância média calculada para cada variável latente foi superior a 0,5, ratificando a validação convergente. A análise confirmatória também foi validada, com valores de confiabilidade composta (CR) e alfa de Cronbach para cada variável latente superior a 0,7. A Tabela 3 apresenta as principais variáveis para validação convergente e análise de confiabilidade. Segundo Hair Jr et al. (2013), quando os valores das cargas externas estiverem entre 0,4 e 0,7, os indicadores devem ser verificados individualmente. OTM6, INO1, INO3, DCF2, DCF3, DCF4 e ISG5, que apresentaram valores abaixo de 0,6, e sua remoção não apresentou variação significativa na AVE e na confiabilidade composta, indicando sua baixa participação na explicação das variáveis latentes às quais pertencem, e por esse motivo foram desconsiderados na análise dos dados.

Tabela 2

Validação discriminante

\begin{tabular}{cccccccc}
$\begin{array}{c}\text { Variável } \\
\text { latente }\end{array}$ & OTM & INO & DCF & ISG & UPC & FUP & IOB \\
OTM & 0.853 & & & & & & \\
INO & 0.534 & 0.790 & & & & & \\
DCF & -0.580 & -0.553 & 0.739 & & & & \\
ISG & -0.648 & -0.429 & 0.721 & 0.750 & & & \\
UPC & 0.602 & 0.438 & -0.601 & -0.551 & 0.876 & & \\
FUP & 0.104 & 0.363 & -0.056 & 0.004 & 0.270 & 0.918 & \\
IOB & 0.726 & 0.699 & -0.706 & -0.686 & 0.735 & 0.245 & 0.968 \\
\hline
\end{tabular}

Fonte: Os autores. 
Tabela 3

Principais variáveis para validação convergente e análise de confiabilidade

\begin{tabular}{|c|c|c|c|c|c|}
\hline Variável latente & Indicador & $\begin{array}{l}\text { Carga } \\
\text { externa }\end{array}$ & $\begin{array}{l}\text { Variância } \\
\text { média extraída } \\
\text { (AVE) }\end{array}$ & $\begin{array}{l}\text { Confiabilidade } \\
\text { composta (CR) }\end{array}$ & $\begin{array}{l}\text { Alfa de } \\
\text { Cronbach }\end{array}$ \\
\hline \multirow{6}{*}{ Otimismo } & OTM1 & 0.873 & \multirow{6}{*}{0.727} & \multirow{6}{*}{0.930} & \multirow{6}{*}{0.909} \\
\hline & OTM2 & 0.856 & & & \\
\hline & OTM3 & 0.848 & & & \\
\hline & OTM4 & 0.794 & & & \\
\hline & OTM5 & 0.889 & & & \\
\hline & OTM6* & 0.533 & & & \\
\hline \multirow{7}{*}{ Inovatividade } & INO1* & 0.497 & \multirow{7}{*}{0.624} & \multirow{7}{*}{0.892} & \multirow{7}{*}{0.849} \\
\hline & INO2 & 0.678 & & & \\
\hline & INO3* & 0.443 & & & \\
\hline & INO4 & 0.880 & & & \\
\hline & INO5 & 0.856 & & & \\
\hline & INO6 & 0.773 & & & \\
\hline & INO7 & 0.745 & & & \\
\hline \multirow{8}{*}{ Desconforto } & DCF1 & 0.600 & \multirow{8}{*}{0.546} & \multirow{8}{*}{0.855} & \multirow{8}{*}{0.796} \\
\hline & DCF2* & 0.283 & & & \\
\hline & DCF3* & 0.507 & & & \\
\hline & DCF4* & 0.322 & & & \\
\hline & DCF5 & 0.789 & & & \\
\hline & DCF6 & 0.861 & & & \\
\hline & DCF7 & 0.766 & & & \\
\hline & DCF8 & 0.650 & & & \\
\hline \multirow{8}{*}{ Insegurança } & ISG1 & 0.837 & \multirow{8}{*}{0.563} & \multirow{8}{*}{0.899} & \multirow{8}{*}{0.871} \\
\hline & ISG2 & 0.629 & & & \\
\hline & ISG3 & 0.733 & & & \\
\hline & ISG4 & 0.636 & & & \\
\hline & ISG5* & 0.368 & & & \\
\hline & ISG6 & 0.675 & & & \\
\hline & ISG7 & 0.833 & & & \\
\hline & ISG8 & 0.867 & & & \\
\hline \multirow{5}{*}{$\begin{array}{l}\text { Utilidade } \\
\text { percebida }\end{array}$} & UPC1 & 0.760 & \multirow{5}{*}{0.767} & \multirow{5}{*}{0.943} & \multirow{5}{*}{0.923} \\
\hline & UPC2 & 0.944 & & & \\
\hline & UPC3 & 0.890 & & & \\
\hline & UPC4 & 0.893 & & & \\
\hline & UPC5 & 0.881 & & & \\
\hline \multirow{4}{*}{$\begin{array}{l}\text { Facilidade de uso } \\
\text { percebida }\end{array}$} & FUP1 & 0.860 & \multirow{4}{*}{0.843} & \multirow{4}{*}{0.956} & \multirow{4}{*}{0.938} \\
\hline & FUP2 & 0.943 & & & \\
\hline & FUP3 & 0.910 & & & \\
\hline & FUP4 & 0.957 & & & \\
\hline \multirow{2}{*}{$\begin{array}{l}\text { Intenção de uso } \\
\text { do Open Banking }\end{array}$} & IOB1 & 0.969 & \multirow{2}{*}{0.938} & 0968 & 0933 \\
\hline & IOB2 & 0.968 & & 0.968 & 0.933 \\
\hline
\end{tabular}

Nota: (Indicadores seguidos por * foram desconsiderados na análise, por apresentarem cargas externas inferiores a 0.6)

Fonte: Os autores. 
A Tabela 4 apresenta os resultados para as hipóteses do modelo. Os resultados sugerem que o Otimismo (OTM) contribui significativamente para a compreensão da Utilidade Percebida (UPC) ( $\beta=$ 0.343) do Open Banking. Por outro lado, sua influência sobre a Facilidade de Uso Percebida (FUP) não foi verificada. Inovatividade (INO) não apresentou influência sobre a UPC, mas atua como preditora da FUP ( $\beta=0.485)$. O Desconforto (DCF) atua negativamente $(\beta=-0.349)$ na compreensão da UPC, mas não tem relação relevante com a FUP. Por fim, se $p<0,1$ for aceito, a Insegurança (ISG) atua negativamente ( $\beta=-0.108$ ) sobre a UPC, mas não tem influência significativa sobre a FUP.

\section{Tabela 4}

\section{Resultados para o Modelo Estrutural}

$\begin{array}{cccccc}\text { Hipótese } & \text { Relação } & \begin{array}{c}\text { Coeficiente de } \\ \text { caminho }(\beta)\end{array} & \text { Testet } & \text { Valor-p } & \\ \mathrm{H}_{1} & \text { OTM } \rightarrow \text { UPC } & 0.343 & 4.710 & <0.001 & \text { Suportada } \\ \mathrm{H}_{2} & \text { OTM } \rightarrow \text { FUP } & -0.007 & 0.094 & 0.925 & \text { Não suportada } \\ \mathrm{H}_{3} & \text { INO } \rightarrow \text { UPC } & -0.072 & 0.649 & 0.517 & \text { Não suportada } \\ \mathrm{H}_{4} & \text { INO } \rightarrow \text { FUP } & 0.485 & 3.707 & <0.001 & \text { Suportada } \\ \mathrm{H}_{5} & \text { DCF } \rightarrow \text { UPC } & -0.351 & 3.316 & 0.001 & \text { Suportada } \\ \mathrm{H}_{6} & \text { DCF } \rightarrow \text { FUP } & 0.122 & 0.727 & 0.468 & \text { Não suportada } \\ \mathrm{H}_{7} & \text { ISG } \rightarrow \text { UPC } & -0.108 & 1.834 & 0.067 & \text { Suportada } \\ \mathrm{H}_{8} & \text { ISG } \rightarrow \text { FUP } & 0.119 & 0.786 & 0.432 & \text { Não suportada } \\ \mathrm{H}_{9} & \text { FUP UPC } & 0.241 & 2.987 & 0.003 & \text { Suportada } \\ \mathrm{H}_{10} & \text { UPC } \rightarrow \text { IOB } & 0.721 & 17.026 & <0.001 & \text { Suportada } \\ \mathrm{H}_{11} & \text { FUP } \rightarrow \text { IOB } & 0.050 & 1.062 & 0.289 & \text { Não suportada }\end{array}$

Fonte: Os autores.

Em resumo, OTM apresentou influência positiva sobre UPC, enquanto DCF e ISG apresentaram influência negativa. Esses resultados confirmam a literatura e mostram que a percepção de utilidade por parte dos usuários é afetada pelo seu otimismo em relação à tecnologia. Por outro lado, o desconforto e a insegurança afetam negativamente a utilidade percebida, o que pode ser um aspecto crítico no caso do Open Banking, já que a sensação de insegurança pode ser bastante aumentada por episódios de vazamento de informações. Em relação à Utilidade percebida, o único resultado que não confirmou a literatura foi o relacionado a INO, que não apresentou influência. Este resultado precisa ser verificado em pesquisa futura.

Em relação à FUP, somente INO apresentou influência, as hipóteses relacionadas a OTM, DCF e ISG não foram confirmadas. A relação entre inovatividade e percepção de facilidade de uso foi encontrada na literatura, mas a falta de influência de otimismo, desconfiança e insegurança contradiz resultados anteriores. Isso pode ser explicado pelo fato de que o Open Banking está em fase de estudos e ainda é pouco conhecido do público, e muitos respondentes tiveram contato com o Open Banking pela descrição contida no questionário. Nesse caso, é razoável supor que a percepção de facilidade de 
uso fique prejudicada, pois o respondente não tem uma referência de como a tecnologia será utilizada. Outros estudos discutiram a adoção de tecnologias já existentes, por exemplo o pagamento por NFC (Shin \& Lee, 2014) ou por sistemas de e-payment (Acheampong et al., 2017), ou o uso de prontuários eletrônicos em celulares por enfermeiras (Kuo et al., 2013), nos quais a percepção de facilidade de uso era auxiliada pelo conhecimento prévio dos respondentes. No caso do estudo da adoção do Open Banking (Sivathanu, 2019), embora sua implantação ainda não esteja finalizada na Índia, o questionário foi administrado precedido por uma explicação e pela exibição de vídeo curto sobre o Open Banking, que pode ter auxiliado a compreensão dos respondentes. Dessa forma, é possível que a falta de relação das variáveis com a percepção de facilidade de uso tenha sido encontrada pelo baixo grau de familiaridade dos respondentes com a tecnologia.

Finalmente, foi constatado que a Facilidade de Uso Percebida (FUP) atua como preditor da Utilidade Percebida (UPC) ( $\beta=0.238$ ), o que confirma diversos autores (Acheampong et al., 2017; Guhr et al., 2013; Shin \& Lee, 2014; Sivathanu, 2019), que também verificaram a relação entre as variáveis latentes. Quanto à compreensão da Intenção de Uso do Open Banking (IOB), foi observado que a UPC atua como forte preditor $(\beta=0.721)$. Por outro lado, notou-se que a FUP não é relevante para o entendimento da IOB, como também observado no estudo de Shin e Lee (2014). Portanto, para os respondentes, as funcionalidades da solução são mais importantes na decisão da adoção do que sua facilidade de uso, ou, como é mais usual atualmente, sua usabilidade. Esse resultado também suaviza o fato de que as variáveis Otimismo, Desconforto e Insegurança não apresentaram relação com a Facilidade de uso percebida, pois essa não apresentou influência na decisão de adoção. A figura 3 exibe o modelo estrutural, com as variáveis latentes estudadas e as relações averiguadas, e os resultados calculados para as relações que apresentaram significância. 
Figura 4

Resultados do modelo PLS-SEM

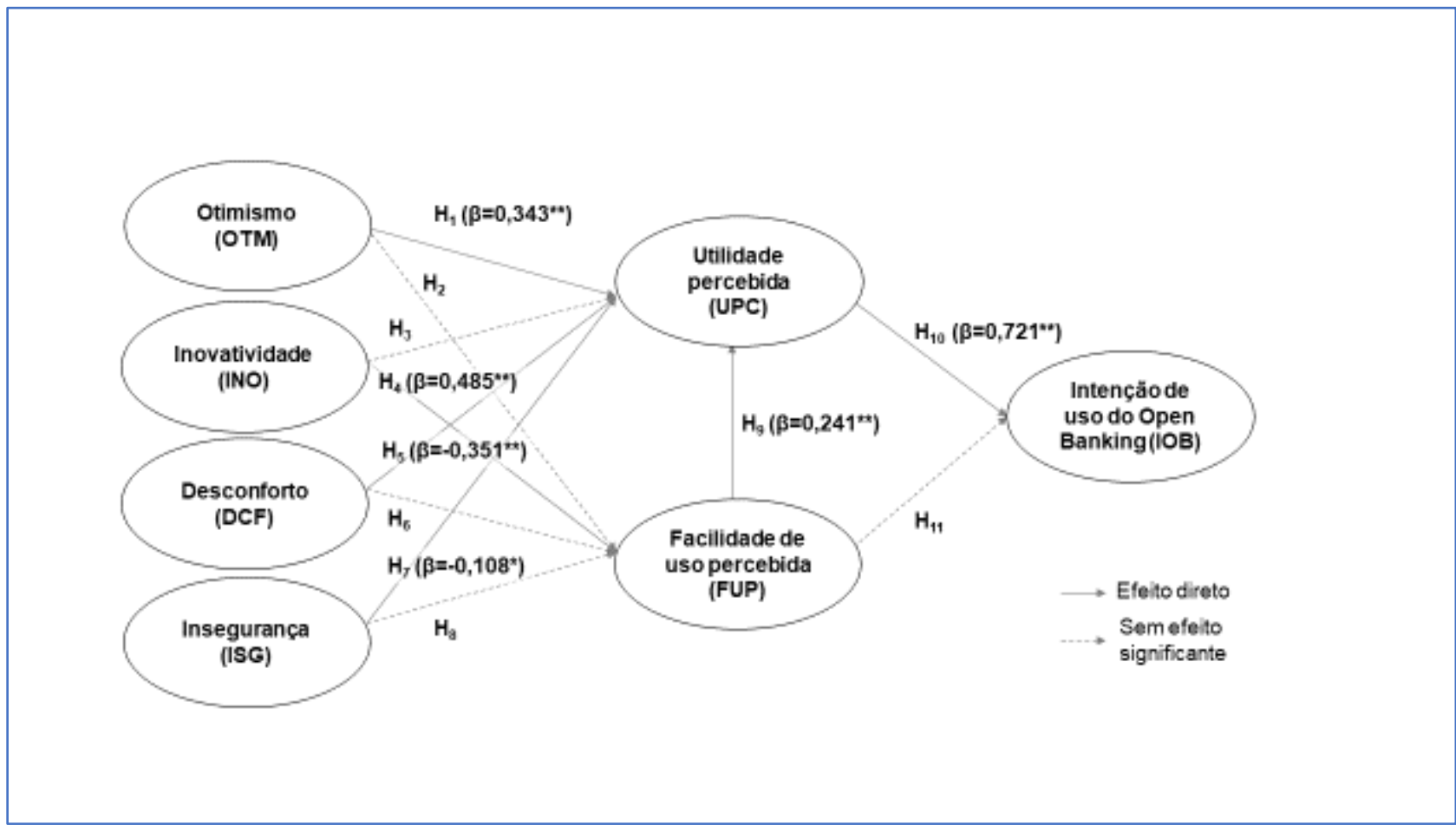

Legenda: * para $p<0,1$ e ** para $p<0,001$

Fonte: Os autores.

\section{Conclusão}

A adoção de novas tecnologias pode reduzir custos e melhorar a qualidade dos serviços, trazendo benefícios para a sociedade, mas pode enfrentar resistência por parte dos usuários, o que pode atrasar ou mesmo inviabilizar sua aplicação. O uso de tecnologia para melhorar a eficiência dos serviços bancários pode reduzir seus custos operacionais, e possibilitar o surgimento de novos prestadores de serviços, aumentando a competição no setor, que é bastante concentrado no país. Com esse objetivo, o Banco Central do Brasil lançou um programa de inovação e modernização, a Agenda BC\#, na qual uma das iniciativas é a implantação do Open Banking, que permite a troca e uso de informações bancárias entre instituições e por novos prestadores de serviços. Espera-se que a adoção de novas tecnologias leve ao aumento da eficiência e da competição, com a redução do spread bancário no país.

Este trabalho fez uma análise da adoção do Open Banking entre jovens universitários, usuários de tecnologias bancárias. Esse público é o potencial alvo da nova tecnologia, e o estudo de sua adoção pode indicar cuidados a serem tomados pela autoridade regulatória. Utilizou-se o TRAM, um modelo que conjuga dois outros bastante utilizados na literatura, o Technology Acceptance Model e o Technology Readiness Index. Estudos sugerem que as variáveis do modelo são sensíveis ao contexto 
tecnológico e à cultura de cada país, e portanto, este trabalho contribui para a literatura pela melhor compreensão do comportamento do modelo e de suas variáveis de influência. Alguns resultados contradizem a literatura existente, e estudos futuros devem verificar a falta de influência do otimismo, desconfiança e insegurança na facilidade de uso percebida. Por outro lado, os dados mostraram que a percepção de facilidade de uso não teve influência na decisão de adoção, o que sugere que, pelo menos no primeiro momento, serão as funcionalidades oferecidas e não a usabilidade, que será decisiva no comportamento dos usuários e na adoção do Open Banking. Isso também sugere que o estudo de tecnologias ainda não disponíveis deve considerar a falta de familiaridade dos respondentes, o que ainda não foi explorado pela literatura. Do ponto de vista de negócios, os resultados indicam que a insegurança é um fator importante para a não-adoção, o que sugere que tanto o Banco Central como os prestadores que utilizarão a tecnologia precisam asseguram ao mercado que não haverá risco de vazamento de dados, o que deve exigir esforço de orientação e comunicação. O tamanho da amostra é uma limitação deste trabalho. Além disso, pelo estágio de implantação do Open Banking no país, a amostra foi composta por jovens habituados à tecnologia digital e ao uso de tecnologias bancárias, que teriam mais facilidade de entender a iniciativa. Porém, o perfil da amostra influencia os resultados e mais estudos com outros segmentos demográficos são necessários para uma visão mais aprofundada do assunto.

\section{Referências}

Acheampong, P., Zhiwen, L., Antwi, H. A., Akai, A., Otoo, A., \& Mensah, W. G. (2017). Hybridizing an Extended Technology Readiness Index with Technology Acceptance Model ( TAM ) to Predict E-Payment Adoption in Ghana. American Journal of Multidisciplinary Research, 5(2).

Davis, F. D. (1989). Perceived Usefulness, Perceived Ease of Use, and User Acceptance of Information Technology. MIS Quarterly, 13(3), 319-340. https://doi.org/10.2307/249008

Guhr, N., Loi, T., Wiegard, R., \& Breitner, M. H. (2013). Technology Readiness in Customers ' Perception and Acceptance of M ( obile ) -Payment : An Empirical Study in Finland, Germany, the USA and Japan. In Rainer Alt \& B. Franczyk (Eds.), 11th International Conference on Wirtschafsinformatik (Issue 1, pp. 119-133). University Leipizig.

Hair, J. F., Anderson, R. E., Tatham, R. L., \& Black, W. C. (1995). Multivariate Data Analysis (4th editio). Pearson.

Hair Jr, J. F., Hult, G. T. M., \& Ringle, C. M. (2013). A Primer on Partial Least Squares Structural Equation Modeling (PLS-SEM) (1st ed.). Sage Publications.

Im, I., Hong, S., \& Kang, M. S. (2011). An international comparison of technology adoption: Testing the UTAUT model. Information and Management, 48(1), 1-8. https://doi.org/10.1016/j.im.2010.09.001 
Kuo, K., Liu, C., \& Ma, C. (2013). An investigation of the effect of nurses ' technology readiness on the acceptance of mobile electronic medical record systems. BMC Medical Informatics and Decision Making, 13(1), 1. https://doi.org/10.1186/1472-6947-13-88

Lam, S. Y., Chiang, J., \& Parasuraman, A. (2008). The effects of the dimensions of technology readiness on technology acceptance: An empirical analysis. Journal of Interactive Marketing, 22(4), 1939. https://doi.org/10.1002/dir.20119

Legris, P., Ingham, J., \& Collerette, P. (2003). Why do people use information technology? A critical review of the technology acceptance model. Information and Management, 40(3), 191-204. https://doi.org/10.1016/S0378-7206(01)00143-4

Lin, C., Shih, H.-Y., \& Sher, P. J. (2007). Integrating Technology Readiness into Technology Acceptance: The TRAM Model. Psychology \& Marketing, 24(7), 641-657. https://doi.org/10.1002/mar

Parasuraman, A. (2000). Technology Readiness Index (Tri): A Multiple-Item Scale to Measure Readiness to Embrace New Technologies. Journal of Service Research, 2(4), 307-320. https://doi.org/10.1177/109467050024001

Parasuraman, A., \& Colby, C. L. (2015). An Updated and Streamlined Technology Readiness Index: TRI 2.0. Journal of Service Research, 18(1), 59-74. https://doi.org/10.1177/1094670514539730

Ramírez-correa, P., Grandón, E. E., \& Rondán-Cataluña, F. J. (2020). Users segmentation based on the Technological Readiness Adoption Index in emerging countries : The case of Chile. Technological Forecasting \& Social Change, 155(April). https://doi.org/10.1016/j.techfore.2020.120035

Rojas-Méndez, J. I., Parasuraman, A., \& Papadopoulos, N. (2017). Demographics, attitudes, and technology readiness: A cross-cultural analysis and model validation. Marketing Intelligence and Planning, 35(1), 18-39. https://doi.org/10.1108/MIP-08-2015-0163

Shin, S., \& Lee, W. (2014). The Effects Of Technology Readiness And Technology Acceptance On NFC Mobile Payment Services In Korea. Journal of Applied Business Research, 30(6), 1615-1626.

Sivathanu, B. (2019). An empirical study on the intention to use open banking in India. Information Resources Management Journal, 32(3), 27-47. https://doi.org/10.4018/IRMJ.2019070102

Sun, S., Lee, P., \& Law, R. (2019). Impact of cultural values on technology acceptance and technology readiness. International Journal of Hospitality Management, 77(June 2018), 89-96. https://doi.org/10.1016/j.ijhm.2018.06.017

Thomas, H., Kimber, A., \& Brown, W. (2019). How regulation is unlocking the potential of open banking in the UK. https://www.ey.com/en_gl/banking-capital-markets/how-regulation-is-unlockingthe-potential-of-open-banking-in-the-uk

Tsikriktsis, N. (2004). A Technology Readiness - Based Taxonomy of Customers. Journal of Service Research, 7(1), 42-52. https://doi.org/10.1177/1094670504266132

Venkatesh, V., Morris, M. G., Davis, G. B., \& Davis, F. D. (2003). User acceptance of information technology: toward a unified view. MIS Quarterly, 27(3), 425-478. 